\title{
Mechanism research and discussion for Electromagnetic projection bidirectional weft insertion Gripper Loom
}

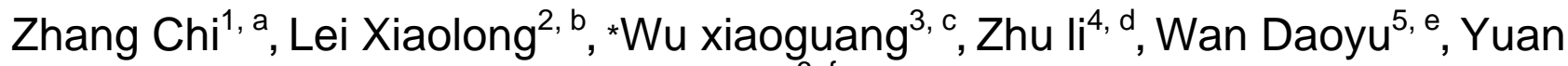 \\ $B 0^{6, f}$ \\ 1, 2, 3,4,5,6 Wuhan Textile University, China \\ ${ }^{*}$ Corresponding Author \\ a55864580@qq.com, b270786321@qq.com, ' $1939394791 @ q q . c o m$, \\ d1293955516@qq.com, ${ }^{\mathrm{e}} 562997653 @ q q . c o m,{ }^{f} 838408681 @ q q . c o m$
}

Keywords: Electromagnetic projection; bidirectional weft insertion; Gripper Loom; Torsion shaft projection Introduction.

Abstract. Based on Electromagnetic projection theory, this paper presents an Electromagnetic bidirectional weft insertion mechanism, and establish its electromagnetism and kinetic model. Based on classical vector Electromagnetic force density model, established projectile Electromagnetic force mechanical model. Find some crucial perimeter about projectile's material and other elements. Discussed the advantages of Electromagnetic projection mechanism over Torsion shaft projection mechanism on the matter of structure and efficiency.

\section{Introduction}

Gripper Loom is the most widely used no shuttle loom. It has the advantages of large weaving width and strong adaptability for variety yarns. However, Because of its higher price and Torsion shaft projection mechanism's low efficiency, limit its wider applicability.

This paper takes Loom Gripper as the research object, presents an Electromagnetic bidirectional weft insertion mechanism. Base on classical Magnetic field Electromagnetic force vector model, establish projectile force density model. By means of analysis on projectile's force field of finite element and calculation, find some crucial perimeter about projectile's material and other elements. Discussed the advantages of Electromagnetic projection mechanism over Torsion shaft projection mechanism on the matter of efficiency.

\section{The principle of Electromagnetic projection}

The basic principle of Electromagnetic projection is live conductor or ferromagnetic object in a magnetic field pushed forward by Electromagnetic force. Electromagnetic projection mechanism mainly consist of coils and projectile. Fig.1 shows the Electromagnetic force directions. When projectile in front of coil, Electromagnetic force pushes projectile move toward coil's center and accelerate projectile. After projectile fly through coil’s center, this force decelerate it.

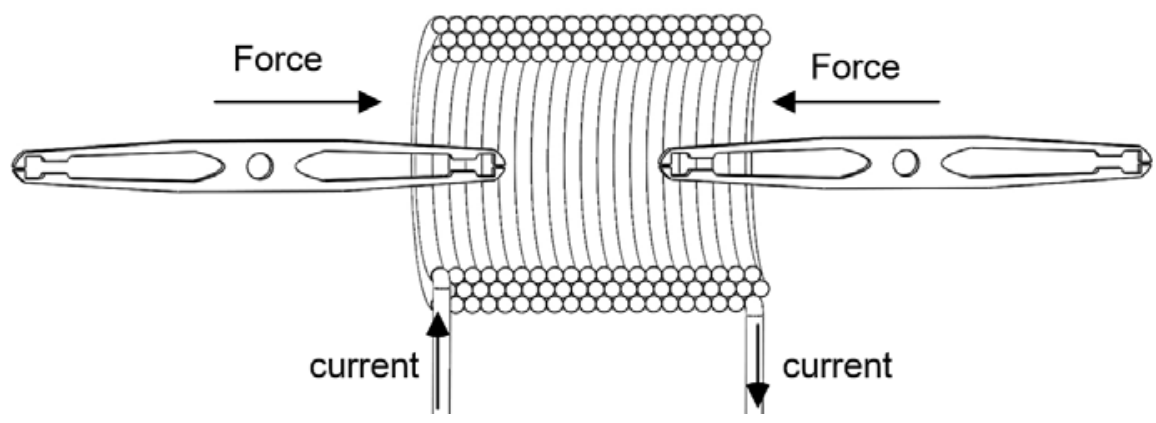

Fig 1 Electromagnetic force direction 
based on simplified excitation model[1], when Linear, Isotropic ferromagnetic projectile In magnetic field, the Electromagnetic force density:

$$
f=J \times B-\frac{1}{2} H^{2} \nabla \mu+\frac{1}{2} \nabla\left[H^{2} \tau \frac{\partial \mu}{\partial \tau}\right]
$$

In which $f$ means Electromagnetic force density vector, $J$ means current density, $B$ means Magnetic induction intensity vector, $H$ means Magnetic field intensity, $\mu$ means the Permeability, $\tau$ means Volume density.

First term in the equation is Lorenz force. The third term represent Magnetostrictive phenomenon. For Linear, Isotropic ferromagnetic media, we can assume that the permeability of the media is only related to its bulk density. 。

After calculated the $\mathrm{X}$ axis force vector, as showed in equation (2), we can conclude the total propulsion force $F_{X}$ in equation (3).

$$
\begin{aligned}
& f_{x}=\frac{\partial}{\partial x}\left[\mu H_{x}^{2}-\frac{H^{2}}{2}\left(\mu-\frac{\partial \mu}{\partial \tau}\right)\right]+\frac{\partial}{\partial y}\left(\mu H_{x} H_{y}\right)+\frac{\partial}{\partial z}\left(\mu H_{x} H_{z}\right) \\
& F_{x}=\int f_{x} d V
\end{aligned}
$$

In which $V$ means projectile's volume.
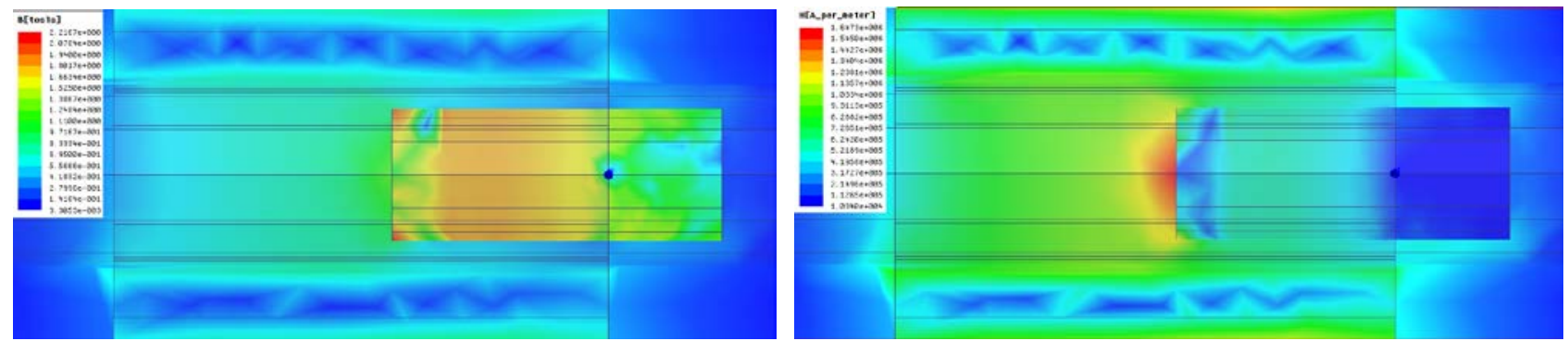

Fig.2 and fig.3 Magnetic induction intensity and magnetic field intensity distribution

According to the formula (1), in order to maximize Electromagnetic force, projectile's Material should have the following properties: 1.low coercive force, it means projectile can be Magnetized or demagnetized in minimum time, avoid deceleration. 2. High magnetic permeability and high saturation magnetic flux density, it means most of Magnetic induction intensity will concentrate inside projectile, increasing Electromagnetic force. Therefore, 1008 steel was selected as projectile's material, it can cover those two things with a certain mechanical strength.

\section{Projectile Kinematics model}

Based on classical Kinematics and Electromagnetic force equation, we can conclude Dynamic motion equation as (4) (5).

$$
\begin{aligned}
& v(t)=v+\int^{t} \frac{\int f_{x} d V}{m} d t \\
& s(t)=s_{0}+\int_{0}^{t} v(t) d t
\end{aligned}
$$

According to simulation result, the projectile's maximum Acceleration of Electromagnetic projection mechanism is around $4000 \mathrm{~m} / \mathrm{s}^{2}$, on the opposite, the torsion shaft mechanism is around 
$18000 \mathrm{~m} / \mathrm{s}^{2}$.compare to torsion shaft mechanism, the new mechanism are more efficient, and less impact force ,longer acceleration time, More adapted to different types of yarn.

\section{Structure of electromagnetic projection mechanism}

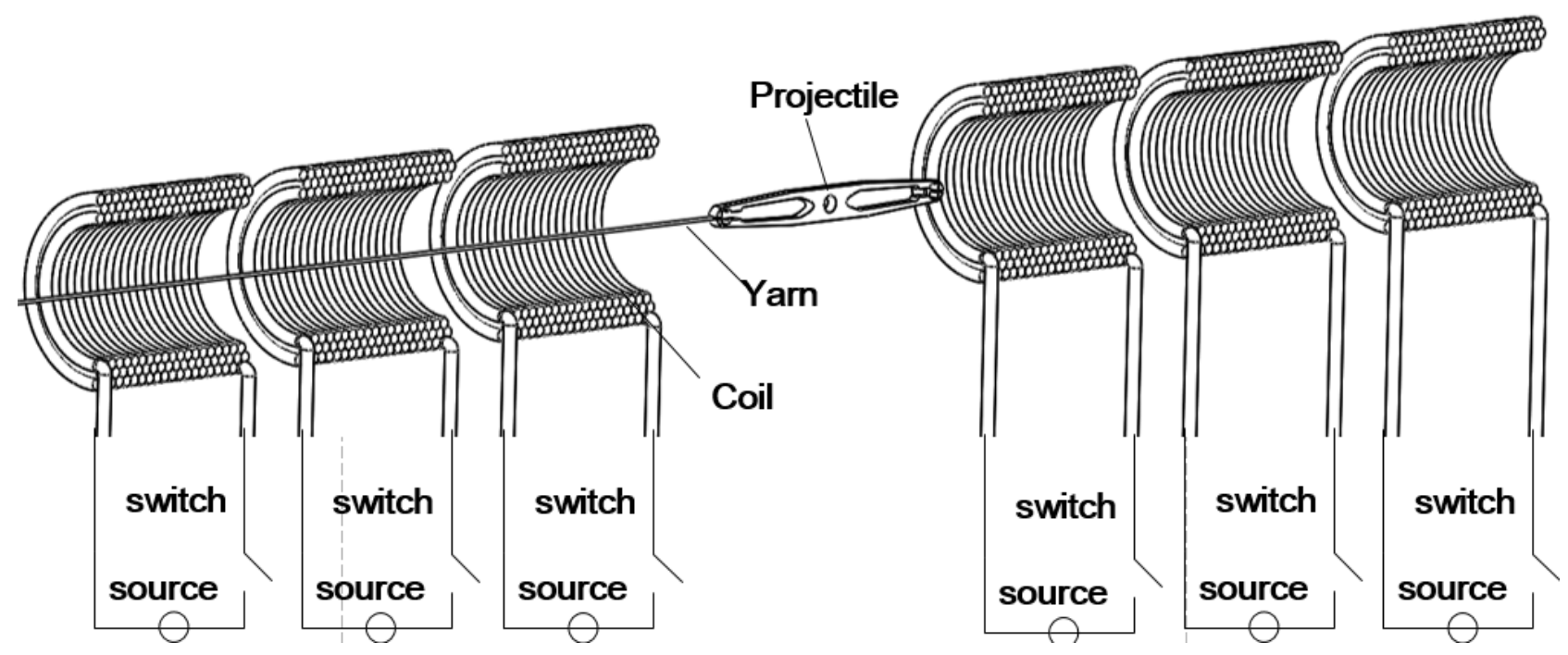

Fig.4 Electromagnetic projection mechanism sketch

Electromagnetic projection model consist of power source, switches, coils and projectile, etc. As shown in fig 4. This mechanism using coil's Electromagnetic force to accelerate projectile. After projectile moved to other side coils, the reversed Electromagnetic force will break projectile and decelerate it. After projectile loaded with yarn, the Electromagnetic force will re-accelerate projectile, and repeat weft insertion process, so that projectile don't have to reverse.

The traditional Gripper Loom using torsion shaft and lever to impact on and accelerate projectile. After one projection process completed, the Hydraulic break mechanism will break the projectile, and convey projectile to projectile lifter, reload and proceed to next weft insertion process [2], as shown in fig.5 and fig.6.

Compared to traditional Gripper Loom, this mechanism omit the torsion shaft and its Transmission device, Hydraulic break mechanism, delivery mechanism, etc. Reduce the impact and vibration of the whole mechanism .Simplified mechanical structure, Improve Gripper Loom's Reliability.

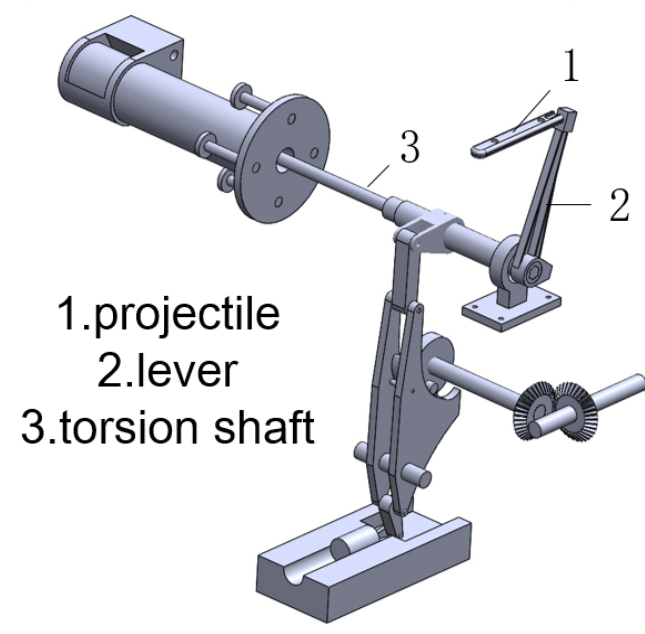

Fig .5 torsion shaft mechanism sketch

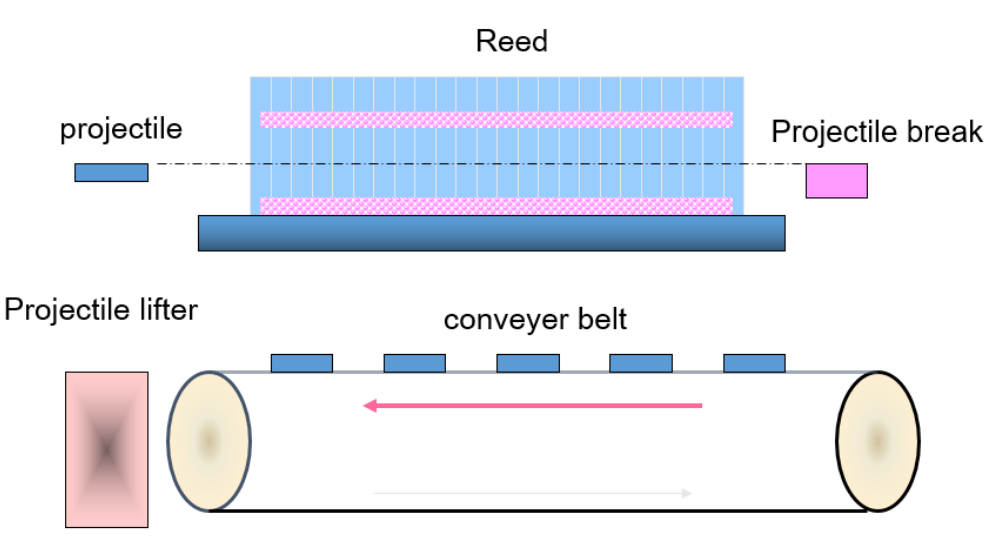

fig. 6 Gripper Loom workflow 


\section{Conclusion}

Due to the asymmetry of the magnetic field distribution, the Electromagnetic force on the projectile are also asymmetric. Compared with axial force, Radial force concentrate on rear side [3], if the stress exceeds allowable stress of materials, and projectile will deformation and cannot continue to weaving. Because of the projectile's Symmetrical design, the front and rear side will take this load alternately. Therefore, when design projectile, the front and rear structure of projectile should be enhance for resist deformation.

In the above introduction, demonstrated that after projectile moving through coil's center, the electromagnetic force will pull the projectile back and decelerate it. If circuit breaker or Synchronous control system cut off the electricity supply when projectile just right at the coil's center, the projectile will continue moving free from deceleration at maximum speed [4]. It means this will require high precision synchronous control system and a complete set of algorithms to cut off circuit right at the time, improve projectile's speed and efficiency.

In addition, generally speaking, capacitance's voltage is proportional to projectile's speed. However, due to the ohmic heat increasing with coil current, in the actual process the projectile's speed is slower than predicted, increase the losses of efficiency[5]. Moreover, different coil structures and capacity value will directly affect mechanism's acceleration time and efficiency [6], the choice of these parameters will be the focus of further research.

\section{Acknowledgement}

The work was supported by National Natural Science Foundation of China (Grant No. 51305309,) and Natural Science Foundation of Hubei Province, (Grant No. 2014CFA099, 2014CFB767)

\section{References}

[1] Gu Xiaoan. A mathematical model of the magnetic force in magnetic materials in a magnetic field [J] ,Noise and vibration control, 2002, 8, (4) .15-30.

[2] ZHENG Xian L. Projectile waving machine [M], Beijing: Publishing House for Textile Industry, 1993.

[3] Zhang Chaowei. Force analysis of the armature of an induction coil gun [J], High Voltage Engineering. 2005, Vol. 31 No. 12.

[4] Guo Wei. Armature deceleration characteristics of synchronous induction coil gun [J]. High Voltage Engineering, 2014, Vol.40, No.4: 1173-1179

[5] Xin Qingwei. Energy conversion efficiency of single stage induction coil gun[J]. Journal of Naval Aeronautical and Astronautical University, 2011, Vol. 26 No.3

[6] Li Sanqun. Influence of structure parameters of the drive coil on the mechanical properties of the armature [J], Micro motor. 2010. Vol.43 No. 8. 